\title{
Modelo Matemático para Planejamento de Produção da Liga SAE 1045 em Fundições
}

\author{
Mathematical Model for Production Planning \\ of Steel Alloys SAE 1045 in Foundries
}

Tiago Moura Kohmann; Antônio C.F Vilela

Laboratório de Siderurgia - LASID-PPGE3M/UFRG: 15021 - 91501-970, Porto Alegre, RS.

e-mail: tiagomk@yahoo.com.br

e-mail: vilela@ufrgs.br

\begin{abstract}
RESUMO
A acurácia da composição química para fabricação de peças fundidas em ligas de aço possui grande importância. Isso se deve à dependência que têm dela as propriedades mecânicas desejadas, o aparecimento de inclusões líquidas ou sólidas e o comportamento diante de tratamentos térmicos. Seu acerto se torna um desafio na etapa de carregamento, quando não se conhece a composição de forma explícita das sucatas e se lida com variadas matérias-primas e preços. Tendo isto em vista, desenvolveu-se em parceria com a fundição Ecoplan Aços Especiais, RS, modelos de eficiência metálica e de carregamento utilizando balanço de massa e conjuntos difusos, juntamente com termodinâmica. Sua implementação foi feita em C\# e a liga testada foi SAE 1045. Os resultados comparados com a literatura se mostraram tecnicamente equivalentes e/ou ainda economicamente melhores. Comparados com a indústria, alcançaram-se corridas com composição química prevista dentro da especificação e com economia para a etapa. Também foram propostos indicadores de custo-benefício e de suprimento de elementos de liga. Foram comparados os indicadores obtidos com os resultados do modelo e da empresa com o método ANOVA. Os indicadores $\zeta$ e $\zeta^{F e}$ foram encontrados menor e maior do que os da empresa, respectivamente, sendo ambos estatisticamente diferentes. $\mathrm{O}$ indicador $\tau$ apresentou equivalência estatística.
\end{abstract}

Palavras-chave: aço, carregamento, fundição, modelo, planejamento.

\section{ABSTRACT}

The accuracy of the chemical composition for the production of steel alloys casting parts has great importance. This is due to the dependence that the desired mechanical properties, the occurrence of liquid or solid inclusions and behavior during heat treatments from this accuracy. Its adjustment becomes a challenge in the loading step, when little is known about the composition of the scraps and one deals with various raw materials and prices. Thus, the authors, in partnership with the casting industry Ecoplan Aços Especiais RS, developed models of metallic yield and of charge using mass and fuzzy sets, along with thermodynamics. Its implementation was made in C\#. Compared with the literature, the findings proved to be technically equivalent and/or better economically. Compared with the industry, the model reached up races for the tested alloy, SAE 1045, with expected chemical composition within specification and with economy for the step. The authors also proposed indicators of cost-benefit and supply of alloying elements. Finally, using ANOVA, this article compares the indicators obtained with the model results and those of the industry. The indicators $\zeta$ and $\zeta^{\mathrm{Fe}}$ were found smaller and larger than those of the industry, respectively, both being statistically different. The indicator $\boldsymbol{\tau}$ showed statistical equivalence.

Keywords: steel, charge, foundry, model, optimization, planning.

\section{INTRODUÇÃO}

A composição química dos aços na siderurgia possui um papel muito importante nas etapas de produção de uma peça fundida. A microestrutura desejada pelo tratamento térmico da peça depende fundamentalmente dos fluxos de calor e da composição química. A microestrutura compõe suas propriedades mecânicas com flutuações dependentes da influência dos elementos de liga. Dessa forma, ao se tratar da engenharia da peça, devem-se manter os olhos também em sua composição e nas implicações subsequentes nos passos de sua manufatura. 
Nas fundições industriais, parte-se como matéria-prima de vários tipos de sucatas, cujo conhecimento da composição química é impreciso. Na etapa de carregamento, busca-se misturar da melhor forma as variadas sucatas adquiridas a diversos preços com o objetivo de melhor compor o banho dentro das especificações, e da forma mais vantajosa economicamente.

Juntamente com as sucatas, usa-se a adição de ferroligas, que visa não apenas à correção química do banho, mas também à desoxidação. De forma que o cálculo para a carregamento necessita levar em conta dois aspectos: o balanço de massa e também a perda metálica dos elementos do banho para a escória, o que é devido às reações de remoção destes elementos que formarão óxidos prejudiciais às propriedades da peça a ser produzida.

Diante da dificuldade de se planejar o carregamento dado o não conhecimento explícito das composições das sucatas, e incluindo-se o aspecto incerto da eficiência metálica para cada elemento, o presente trabalho vem a desenvolver um modelo conjunto entre termodinâmica, balanços de massa e conjuntos difusos para tal etapa. O objetivo principal é desenvolver uma ferramenta que sirva de forma técnica e economicamente eficiente às indústrias de fundição que enfrentam dificuldades no planejamento de suas corridas.

\section{REFERENCIAL TEÓRICO}

Apresenta-se a seguir uma breve conceituação da obtenção da concentração de oxigênio no banho, da eficiência metálica e de conjuntos difusos, de forma a ser possível melhor entender o desenvolvimento do modelo matemático proposto.

\subsection{Cálculo do Oxigênio no Banho}

A concentração inicial de oxigênio no banho pode ser calculada considerando-o em equilíbrio com cada elemento $M$, de acordo com a reação (1), e tomando-se seu valor mais elevado. A constante de equilíbrio $K$ genérica para a reação (1) se encontra descrita na equação (2), onde os valores de seus coeficientes $A[1]$ e $B\left[K^{-1}\right]$ se encontram em CARVALHO et al[1].

$n_{i} \underline{M}+n_{O, i} \underline{O}=M_{n_{i}} O_{n_{O, i}}$

$K=10^{(A+B * T)}=\frac{a_{M_{n_{i}} O_{n_{O, i}}}}{a_{M}^{n_{i}} a_{O}^{n_{O, i}}}$

Considera-se que as atividades dos óxidos do elemento $M$ são unitárias, que as atividades dos elementos são henrianas com influência dos demais (onde seus coeficientes podem ser igualmente calculados a partir de [1] ), e temperatura de fornada de $T=1723 \mathrm{~K}$.

\subsection{Eficiência Metálica}

Segundo CAMPOS [2], a eficiência metálica do i-ésimo elemento $\varphi_{i}$ [\%] é definida pela razão entre a massa do i-ésimo elemento que não reagiu e sua massa adicionada $m_{a, i}[\mathrm{~kg}]$, sendo sua massa que reage igual a $m_{r, i}[k g]$, conforme (3).

$\varphi_{i}=1-\frac{m_{r, i}}{m_{a, i}}$

\subsection{Conjuntos Difusos}

Um número $\mathbf{x}$ é dito pertencer ao conjunto $\mathbf{A}$ através de uma função de filiação difusa $\boldsymbol{\mu}_{\mathbf{A}}(\mathbf{x})$ se estiver dentro de uma delimitação, conforme TERANO et al[3]]. No presente artigo, a composição química do i-ésimo elemento pertence à liga ou à j-ésima sucata se estiver dentro dos seus limites inferior e superior de especificação, segundo ZIÓLKOWSKI e WRONA [4], como definido na função de filiação quadrada em (4).

$\mu_{j}(i)=\left\{\begin{array}{c}1, \quad \underline{w_{i, j}} \leq w_{i, j} \leq \overline{w_{i, j}} \\ 0, w_{i, j}<\underline{w_{i, j}} \text { ou } \overline{w_{i, j}}<w_{i, j}\end{array}\right.$ 


\section{MATERIAIS E MÉTODOS}

Nesta seção é desenvolvido o modelo matemático em duas partes. Primeiro, desenvolve-se a eficiência metálica. Em seguida, o modelo matemático para o carregamento. Após o desenvolvimento dos modelos, é apresentada a metodologia de validação dos modelos.

\subsection{Desenvolvimento da Eficiência Metálica}

Aplicando um balanço de massa na fase escória, vê-se que a massa do i-ésimo elemento que reage é igual à diferença entre as composições inicial $(i)^{i}\left[\%_{\text {mássico }}\right]$ e final $(i)\left[\%_{\text {mássico }}\right]$ do i-ésimo elemento na escória multiplicada por suas massas inicial $m_{e}^{i}[\mathrm{~kg}] \mathrm{e}$ final $m_{e}[\mathrm{~kg}]$, conforme (5).

$m_{r, i}=m_{e}(i)-m_{e}^{i}(i)^{i}=m_{e}(i)$

Para o i-ésimo elemento, considerar-se-á a reação de oxidação de $n_{i}$ moles do i-ésimo elemento com $n_{O, i}$ moles de oxigênio gerando $1 \mathrm{~mol}$ de seu óxido $\left(i_{n_{i}} O_{n_{O, i}}\right)$, descrita em (6).

$n_{i} \underline{i}+n_{O, i} \underline{O}=\left(i_{n_{i}} O_{n_{O, i}}\right)$

Há uma relação estequiométrica entre a massa do i-ésimo elemento e a massa de oxigênio $m_{r, o}[\mathrm{~kg}]$ que reagem, que pode ser vista em (7), onde $\bar{M}_{i}\left[\frac{\mathrm{kg}}{\mathrm{kgmol}}\right]$ e $\bar{M}_{O}\left[\frac{\mathrm{kg}}{\mathrm{kgmol}}\right]$ são as massas molares respectivas.

$\frac{n_{O, i}}{\bar{M}_{i}} m_{r, i}=\frac{n_{i}}{\bar{M}_{O}} m_{r, O}$

A massa de oxigênio que reage é dada pela diferença entre as frações mássicas inicial e final de oxigênio, multiplicada pela carga da fornada $m_{b}[\mathrm{~kg}]$, segundo (8).

$m_{r, O}=m_{b}\left([O]^{i}-[O]\right)$

Além disso, a massa de oxigênio que reage com o i-ésimo elemento necessita ser multiplicada pela fração da massa de oxigênio presente na escória que é proveniente de seu óxido. Assim, se obtém na equação (9) a massa do i-ésimo elemento que reage.

$m_{r, i}=m_{b}\left([O]^{i}-[O]\right) \frac{\bar{M}_{i}}{\bar{M}_{O}} \frac{n_{O, i}}{n_{i}} \frac{n_{O, i}(i)}{\sum_{i^{\prime}} n_{O, i}\left(i^{\prime}\right)}$

Para o caso de um óxido gasoso, usa-se a equação (10) para o cálculo da pressão gerada do óxido $P_{O, i}$, convertida então para a massa através da equação (11) com auxílio da equação dos gases ideais, onde $V[L]$ é o volume de um mol e $K_{i}$ é a constante de reação de formação do gás.

$K_{i}=\frac{P_{O, i}}{[i]^{n_{i}}[O]^{n_{O, i}}}$

$m_{r, i}=\frac{K_{i}[i]^{n_{i}}[O]^{n_{O, i}} V \overline{M_{O, i}}}{n_{i} R T}$

A massa do i-ésimo elemento adicionada é obtida pela soma das multiplicações entre as frações mássicas $w_{i, j}\left[\%_{\text {mássico }}\right]$ e $v_{i, k}\left[\%_{\text {mássico }}\right]$ e as cargas das j-ésimas sucatas $s_{j}[k g]$ e k-ésimas adições $a_{k}[\mathrm{~kg}]$, conforme a equação (12).

$m_{a, i}=\sum_{j} s_{j} w_{i, j}+\sum_{k} a_{k} v_{i, k}$ 


\subsection{Desenvolvimento do Modelo Matemático para Carregamento}

Conforme SAKALLI e BAYKOÇ [5] e RONG e LAHDELMA [6], deseja-se minimizar a função objetivo $F[\$]$ que representa o custo para o carregamento do forno com as j-ésimas sucatas e k-ésimas adições, descrita matematicamente na equação (13), onde $c_{j}\left[\frac{\$}{\mathrm{~kg}}\right]$ e $d_{k}\left[\frac{\$}{\mathrm{~kg}}\right]$ são os custos por quilograma de cada sucata e adição.

$F=\sum_{j} s_{j} c_{j}+\sum_{k} a_{k} d_{k}$

Para a obtenção das condições de contorno da função objetivo, faz-se uso na equação (14) de um balanço de massa parcial do i-ésimo elemento na fase do banho $[i]\left[\%_{\text {mássico }}\right]$.

$\sum_{j} s_{j} w_{i, j}+\sum_{k} a_{k} v_{i, k}=[i] m_{b}$

Acrescenta-se à equação (14) as condições provenientes da função difusa de filiação (4), conforme [4]. Isto é, se obtém na equação (15) que a soma dos limites inferiores de composição das j-ésimas sucatas $w_{i, j}\left[\%_{\text {mássico }}\right]$ com as composições das k-ésimas adições necessita ser maior ou igual ao limite mínimo de especificação da liga $[i]$ [\% $\left.\%_{\text {mássico }}\right]$. De forma similar, a soma dos limites superiores de composição das $\mathrm{j}$ ésimas sucatas $\overline{w_{i, j}}\left[\% \%_{\text {mássico }}\right]$ com as composições das k-ésimas adições necessita ser menor ou igual ao limite máximo de especificação da liga $\overline{[i]}\left[\%{ }_{\text {mássico }}\right]$.

$$
\left\{\begin{array}{l}
\sum_{j} s_{j} \underline{w_{i, j}}+\sum_{k} a_{k} v_{i, k} \geq \underline{[i]} m_{b} \\
\sum_{j} s_{j} \overline{w_{i, j}}+\sum_{k} a_{k} v_{i, k} \leq \overline{[i]} m_{b}
\end{array}\right.
$$

Às condições de contorno em (15) se multiplica as eficiência metálicas inferior $\varphi_{i}$ [\%] e superior $\overline{\varphi_{i}}[\%]$ do i-ésimo elemento obtidas em (3), chegando-se às condições de contorno em (16).

$$
\left\{\begin{array}{l}
\left(\sum_{j} s_{j} \underline{w_{i, j}}+\sum_{k} a_{k} v_{i, k}\right) \underline{\varphi_{i}} \geq \underline{[i]} m_{b} \\
\left(\sum_{j} s_{j} \overline{w_{i, j}}+\sum_{k} a_{k} v_{i, k}\right) \overline{\varphi_{i}} \leq \overline{[i]} m_{b}
\end{array}\right.
$$
satisfeita.

Além destas condições, há também a condição do balanço de massa global (17) que necessita ser

$$
\sum_{j} s_{j} \phi_{j}+\sum_{k} a_{k} \psi_{k} \geq m_{b}
$$

As eficiências médias das sucatas e adições $\phi_{j}[\%]$ e $\psi_{k}[\%]$ podem ser calculadas através da soma das eficiências dos elementos que as constituem ponderadas por suas composições.

Este modelo matemático pode ser resolvido com qualquer técnica de programação linear.

\subsection{Metodologia de Validação com a Literatura}

De posse dos dados de [4], realizou-se simulação com as composições e eficiências metálicas disponibilizadas pelos autores, com massa total acrescentada menor ou igual do que $300 \mathrm{~kg}$ (cenário 1) e maior ou igual do que $200 \mathrm{~kg}$ (cenário 2). Todos os custos relativos foram considerados unitários. Os resultados se encontram na seção 4.1 . 


\subsection{Metodologia de Validação com a Prática Industrial}

Foram acompanhados o carregamento e a otimização de 13 corridas da liga SAE 1045, cuja especificação se encontra na Figura 1. As sucatas disponíveis para o carregamento do forno se encontram na Figura 2 e as adições, na Figura 3. Todos os seus valores de custos $\left[\frac{\$}{\mathrm{~kg}}\right]$, determinados pelo quociente entre o investimento de aquisição e a quantidade adquirida, são relativos à Sucata Comum, apresentados na Figura 4. Foram coletadas também 6 amostras de escórias para conhecimento da sua composição aproximada e uso nos modelos. Seus valores se encontram na Figura 5. O uso da ferramenta ANOVA se encontra descrito no item 9, Anexos, de acordo com MONTGOMERRY [7].

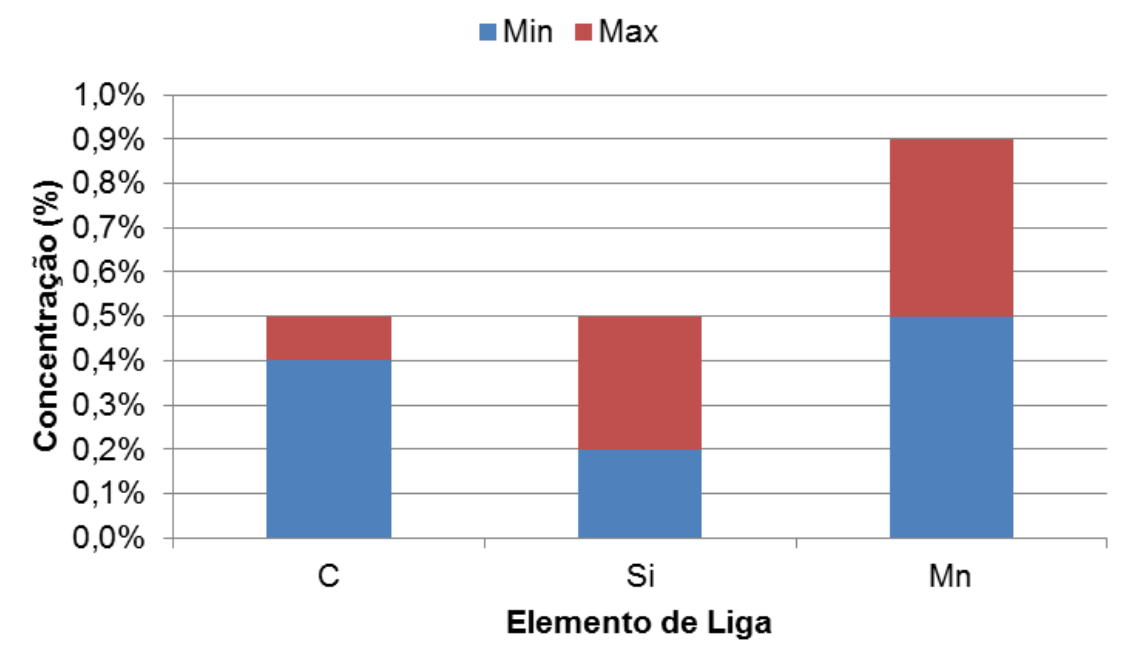

Figura 1: Composição Química da Liga SAE 1045 e de sua Sucata de Processo

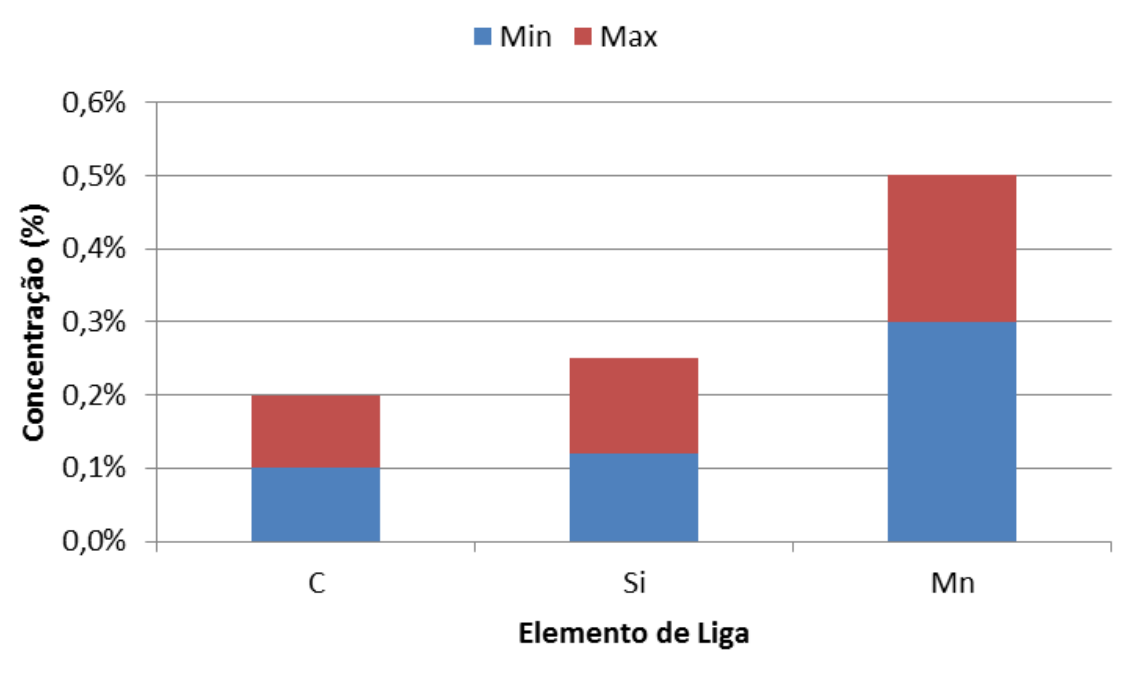

Figura 2: Composição Química da Sucata Comum 




Figura 3: Composição Química das Adições



Figura 4: Custo Relativo das Matérias-Primas

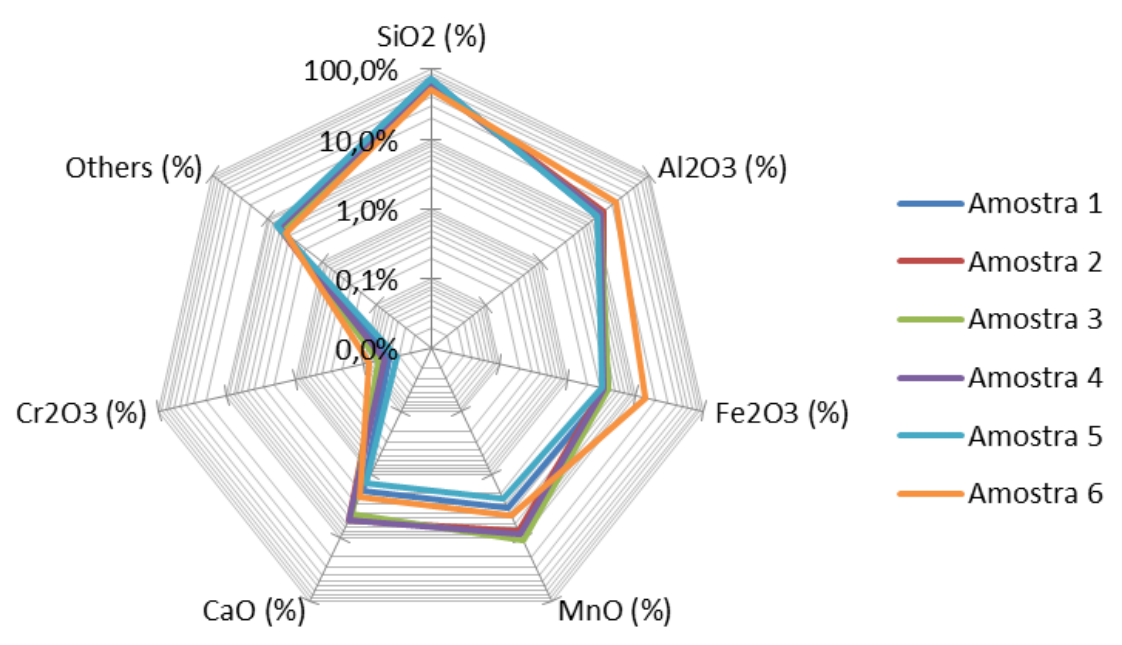

Figura 5: Composição Química das Amostras de Escória

\section{RESULTADOS}

A seguir se encontram os resultados das simulações com dados da literatura e da prática industrial. 


\subsection{Simulação com Dados da Literatura}

Os resultados de [4] e das simulações deste modelo se encontram presentes na Figura 6. Tanto para os resultados da literatura quanto para o cenário 1, apesar de as cargas individuais da Sucata 2 e da Sucata 4 diferiram levemente, as massas totais adicionadas foram as mesmas, além de o custo total de carregamento ser igual para ambos, no valor de $\$ 0,2305 / \mathrm{kg}$. No cenário 2, a carga total foi $78,9 \mathrm{~kg}$ menor do que a literatura, 14,3 kg maior para a Sucata 2 e 93,2 kg menor para a Sucata 4 e o custo total de carregamento foi de $\$ 0,1808 / \mathrm{kg}$. As concentrações finais mínima e máxima alcançadas também se encontram na Figura 2. Nota-se que ambos os cenários simulados permaneceram extremamente próximos das concentrações finais de [4]

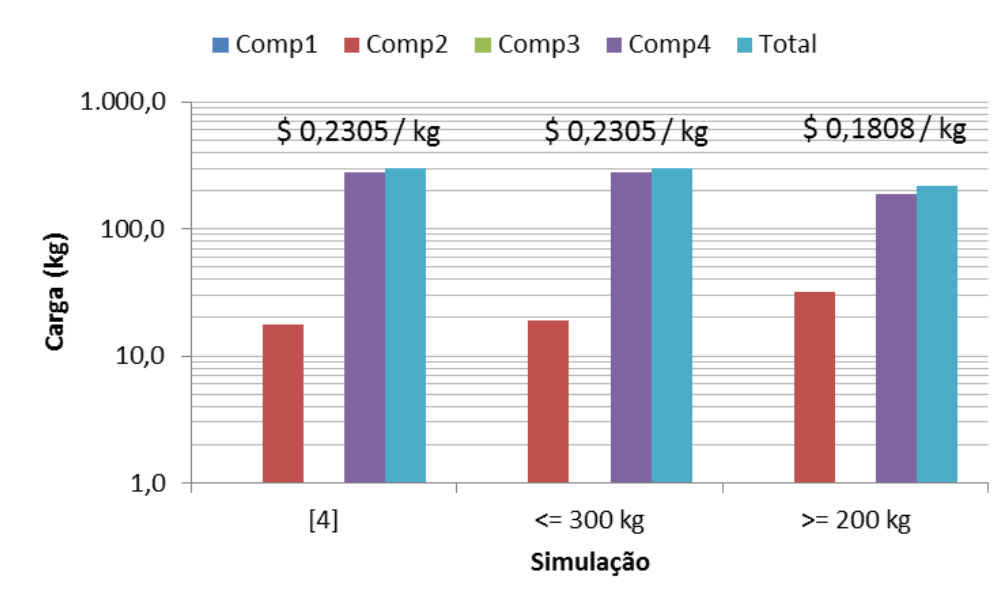

Figura 6: Cargas das Sucatas e Custo Relativo para os Resultados em [4] e os Cenários Simulados

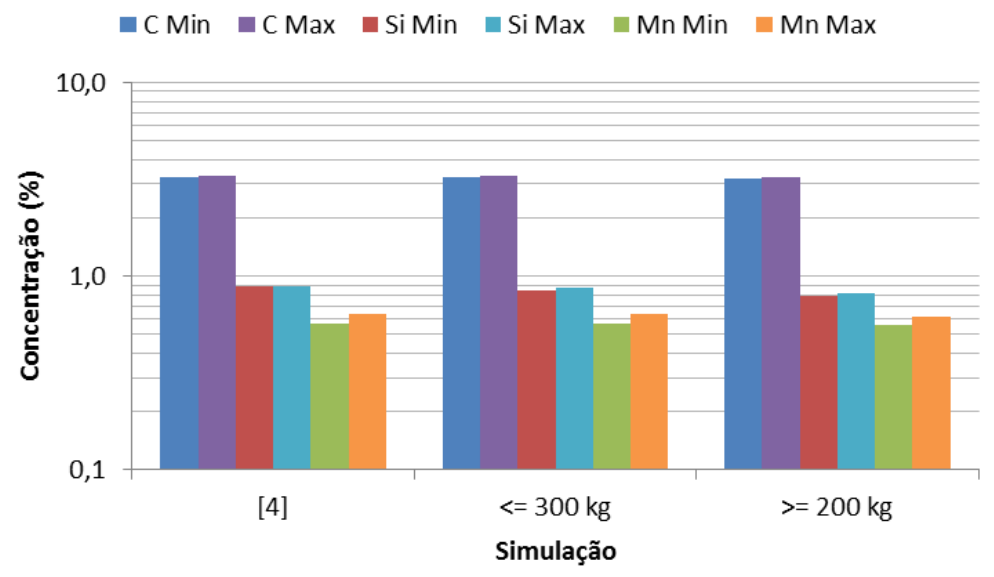

Figura 7: Composições Químicas Finais Mínima e Máxima para os Resultados em [4] e os Cenários Simulados

\subsection{Simulação com Dados da Indústria}

As informações das práticas de carregamento da empresa se encontram na Figura 5. As composições químicas alcançadas, na Figura 6. 


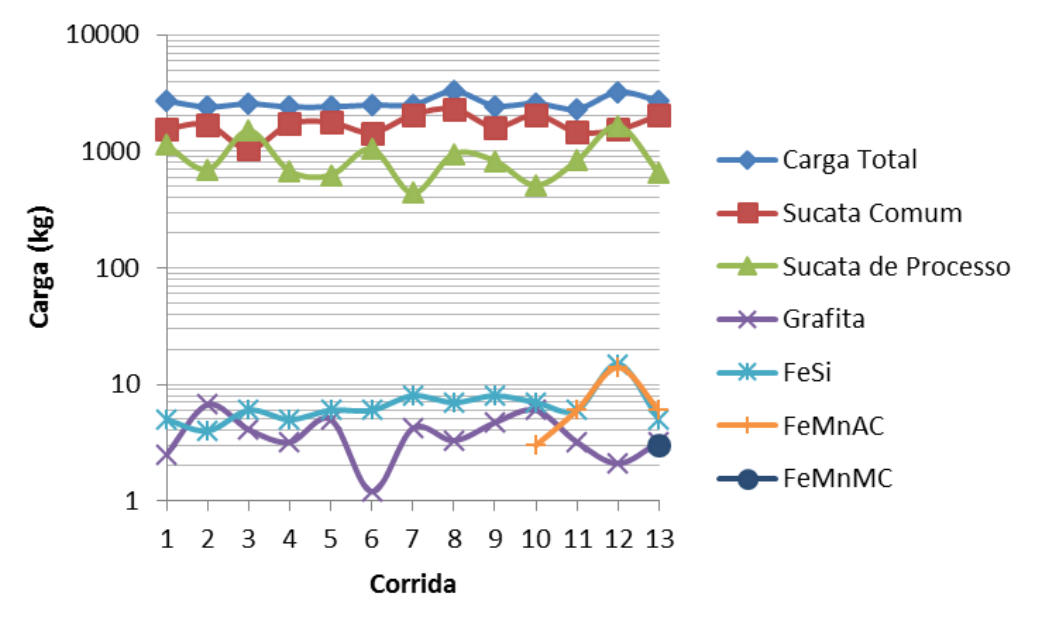

Figura 8: Carregamentos Realizados pela Empresa

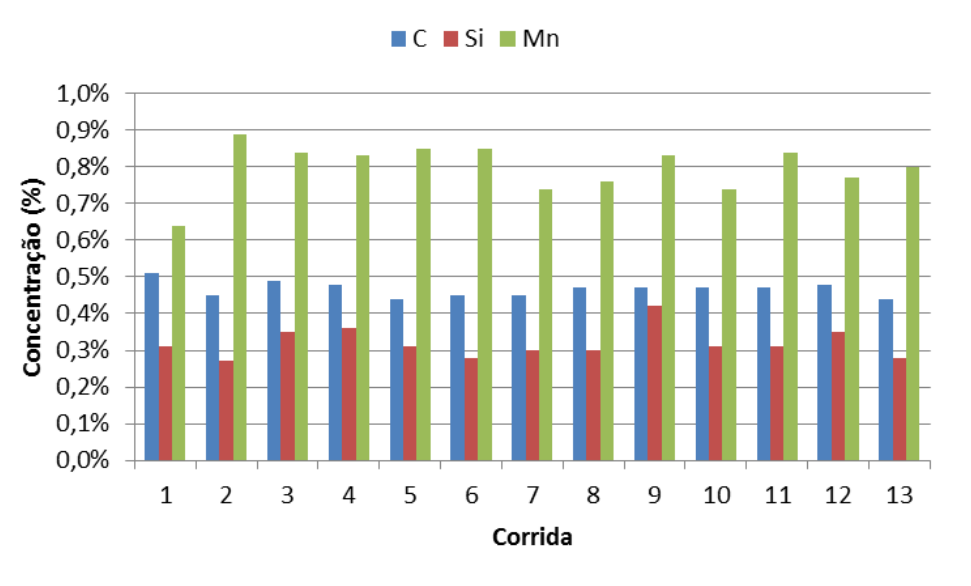

Figura 9: Composição Química Alcançada ao Término de cada Corrida

Na Figura 8, se encontram as eficiências metálicas mínimas e máximas médias para cada elemento, de acordo com a prática industrial. Seus valores médios junto com o desvio padrão são de $(99,04 \pm 3,39) \%$ para o carbono, $(72,18 \pm 11,24) \%$ para o silício e $(99,07 \pm 3,34) \%$ para o manganês.

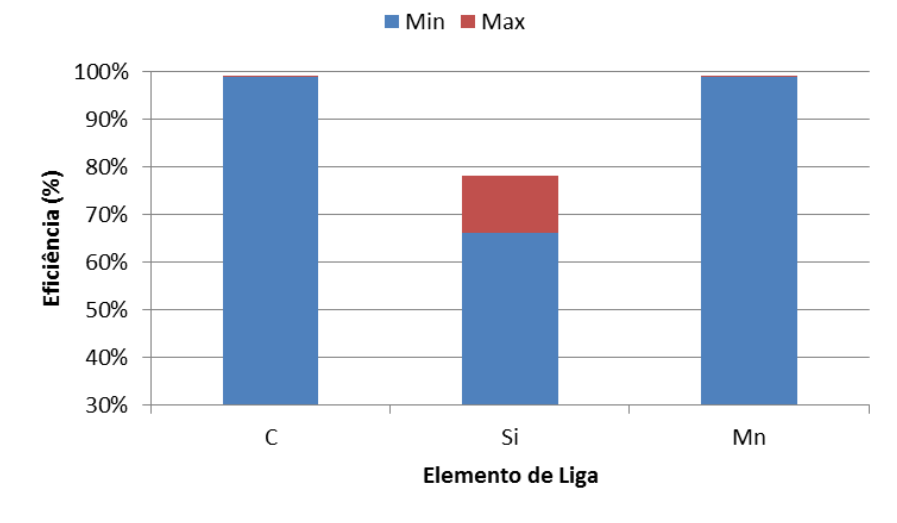

Figura 10: Eficiência Metálica Industrial

O custo relativo de cada corrida se encontra na Figura 9. 




Figura 11: Custo Relativo Industrial

De posse do carregamento realizado, empregou-se o modelo aqui desenvolvido visando um carregamento mais economicamente eficiente. Dessa forma, gerou-se o gráfico da Figura 10, que dispõe dos resultados do modelo deste artigo para a etapa de carregamento. Na Figura 11, se encontram as composições químicas médias previstas de acordo com o modelo. Seus desvios padrões são $\leq 10^{-3} \%$.

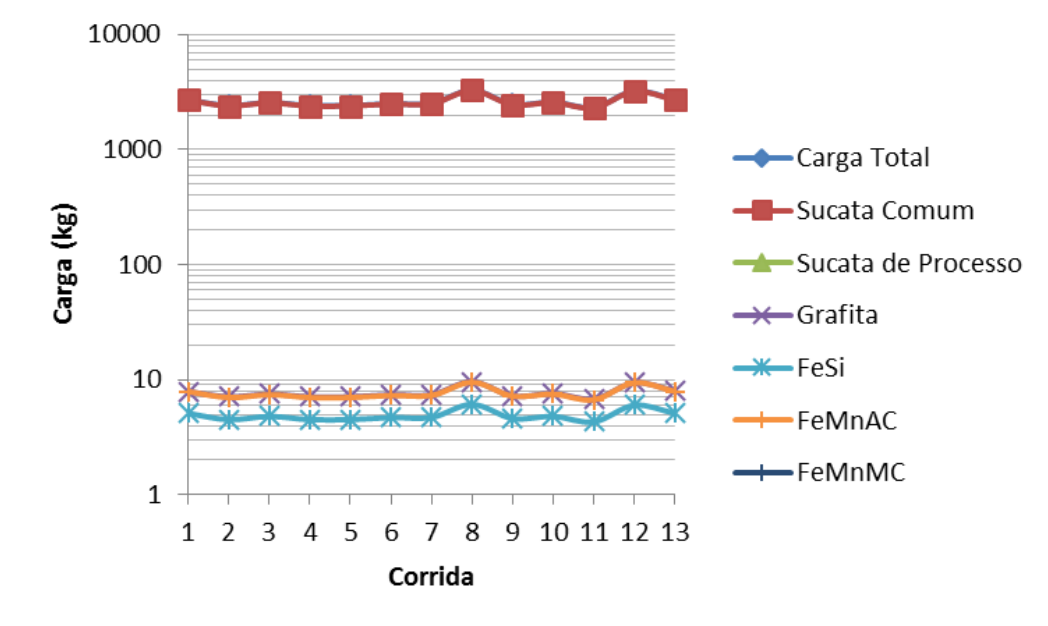

Figura 12: Resultado do Carregamento Obtido com o Modelo

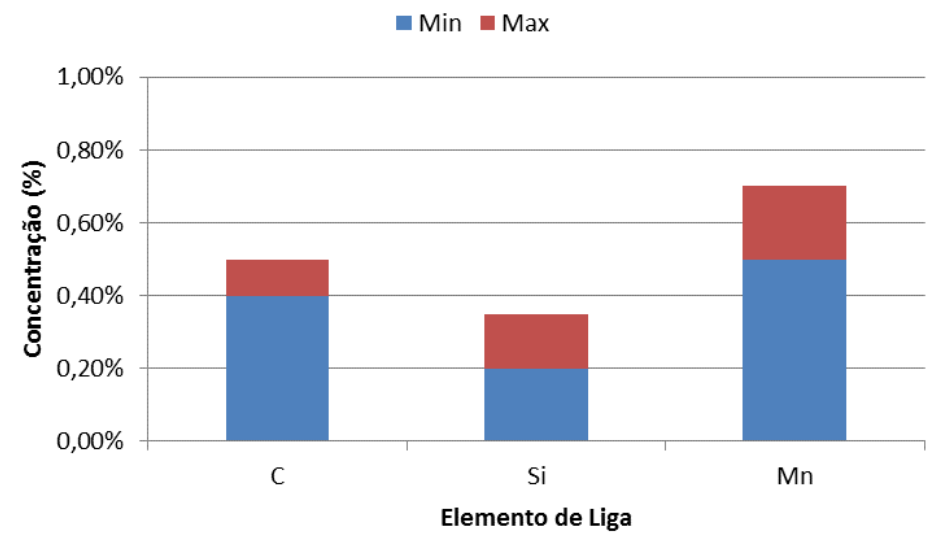

Figura 13: Composição Química Média Obtida com o Modelo

As eficiências metálicas obtidas com o modelo se encontram na Figura 12. 


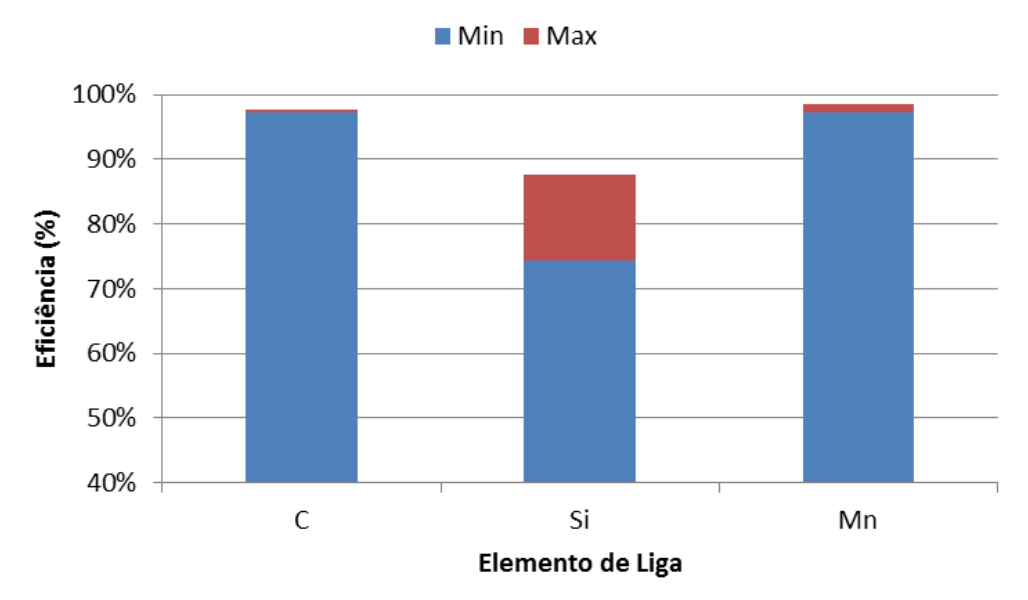

Figura 14: Eficiência Metálica Obtida com o Modelo



Figura 15: Custo Relativo Obtido com o Modelo

\section{DISCUSSÃO}

A seguir, são propostos os indicadores de custo-benefício $\zeta_{j}\left[\frac{\$}{k g_{\text {elem liga }}}\right]$ e $\zeta_{j}^{F e}\left[\frac{\$}{k g_{F e}}\right]$, definidos de acordo com as equações (18) e (19).

$\zeta_{j}=\frac{c_{j}}{\sum_{i \neq F e} w_{i, j}}$

$\zeta_{j}^{F e}=\frac{c_{j}}{w_{F e, j}}$

Elas relacionam a razão entre o custo por $\mathrm{kg}$ da matéria-prima com, respectivamente, a fração mássica referente aos elementos de liga e ao ferro. Segundo elas, são avaliadas as relações de custo e benefício de cada sucata e adição de suprir as necessidades dos elementos da corrida. Quanto menor o seu valor, mais vantajoso economicamente é o seu uso. A Tabela 1 contém seus valores para cada uma das matérias-primas.

Tabela 1: Índices de Custo-Benefício $\zeta_{j}$ e $\zeta_{j}^{F e}$ para as Matérias-Primas 


\begin{tabular}{c|c|c}
\hline Matérias-Primas & $\zeta_{j}$ & $\zeta_{j}^{F e}$ \\
\hline Sucata Comum & 42,134 & 0,40391 \\
Sucata de Processo & 61,497 & 0,88353 \\
Grafita & 2,764 & 539,21569 \\
FeSi & 4,231 & 14,99318 \\
FeMnAC & 3,653 & 16,77852 \\
FeMnMC & 6,765 & 28,03498 \\
\hline
\end{tabular}

De acordo com os resultados da Figura 10, nota-se a predominância unânime do uso de Sucata Comum. Isto é explicado porque seu índice $\zeta_{j}^{F e}$ apresenta a relação mais vantajosa de custo-benefício para o acréscimo de ferro. O aparecimento de Grafita, FeSi e FeMnAC em lugar de Sucata de Processo ou ainda FeMnMC, para o manganês, também é explicado por seus índices $\zeta_{j}$ serem os menores. A Sucata de Retorno não é alternativa economicamente viável devido à sua precificação, tanto para elementos de liga quanto para ferro. Além do mais, seus usos também se limitam entre $30 \%$ e $50 \%$ da carga da fornada, considerando sua quantia em estoque.

Outro índice apresentado é o indicador de suprimento de elementos de liga à fornada em produção, $\tau_{j}^{\text {liga }}[1]$, descrita em (20), que fornece informações sobre a capacidade de cada matéria-prima suprir os elementos necessários, com exceção do ferro. Quanto mais seus valores se aproximarem do valor unitário, mais adequada será a matéria-prima em suprir as necessidades em massa dos elementos de liga da corrida em produção. Seus valores para as matérias-primas se encontram na Tabela 1.

$\tau_{j}=\frac{\sum_{i \neq F e} w_{i, j}}{\sum_{i \neq F e}[i]}$

Tabela 2: Valores do Índice $\tau_{j}$ para as Matérias-Primas

\begin{tabular}{c|c}
\hline Matérias-Primas & $\tau_{j}$ \\
\hline Sucata Comum & 0,6582 \\
Sucata de Processo & 1,0000 \\
Grafita & 64,8128 \\
FeSi & 56,1870 \\
FeMnAC & 57,6474 \\
FeMnMC & 56,1037 \\
\hline
\end{tabular}

Ao se calcularem as médias ponderadas destes indicadores pelas quantias de cada matéria-prima utilizada para cada corrida, e ao se tomar suas médias, obtêm-se os índices médios da Tabela 3, onde foram calculadas as suas médias entre as 13 corridas da liga SAE 1045. Sobre estes mesmos valores, foi realizado o teste ANOVA, onde os resultados da função de probabilidade $F$ são de 102,13 para o indicador $\zeta_{j}, 10773,49$ para o indicador $\zeta_{j}^{F e}$, e 1,05 para o indicador $\tau_{j}$, sendo que o valor tabelado de $F$ é de 4,26. Detalhes do seu cálculo podem ser obtidos no item 9 , Anexos.

Tabela 3: Médias e Desvios Padrões das Médias Ponderadas dos Indicadores do Carregamento

\begin{tabular}{c|c|c|c}
\hline Método & $\overline{\bar{\zeta}}$ & $\overline{\overline{\zeta_{J}}}$ & $\overline{\bar{\tau}_{j}}$ \\
\hline Empresa & $92,17 \pm 4,928$ & $1,06 \pm 0,102$ & $0,882 \pm 0,05091$ \\
Modelo & $77,79 \pm 0,001$ & $4,16 \pm 0,015$ & $0,897 \pm 0,00084$ \\
\hline
\end{tabular}

De acordo com estes resultados, os indicadores $\overline{\overline{\zeta_{J}}}$ e $\overline{\overline{\bar{\zeta}_{J}}}$ são estatisticamente diferentes, mostrando que o 
modelo sugere carregamentos que custam menos com elementos de liga e mais com ferro quando comparado com a metodologia da empresa. Apesar do indicador $\overline{\overline{\zeta_{j}^{F e}}}$ ser menor para a metodologia da empresa, o custo geral dos carregamentos é menor para os resultados deste modelo, demonstrando que a economia nos custos dos elementos de liga compensam gastos a mais com suprimentos de ferro. Eles também demonstram que os elementos de liga possuem um valor econômico intrínseco maior que o ferro. Já o indicador $\overline{\overline{\tau_{j}}}$ apresentou-se estatisticamente igual entre as metodologias da empresa e deste artigo, mostrando que ambos são tecnicamente equivalentes com relação ao suprimento de elementos de liga durante o carregamento.

Considerando a eficiência metálica calculada pelo modelo, seus valores são maiores com relação às eficiências obtidas pela prática empresarial. Isso pode ser explicado pela renovação intencional da escória durante a corrida, acarretando na maior remoção dos elementos de liga através de seus óxidos. Para se estimar as eficiências metálicas simulando a remoção intencional com os valores obtidos pelo modelo, podese elevá-los ao quadrado, estando seus valores demonstrados na Figura 13. Nota-se nela que seus resultados se aproximam dos valores encontrados pela empresa, em especial para o silício.

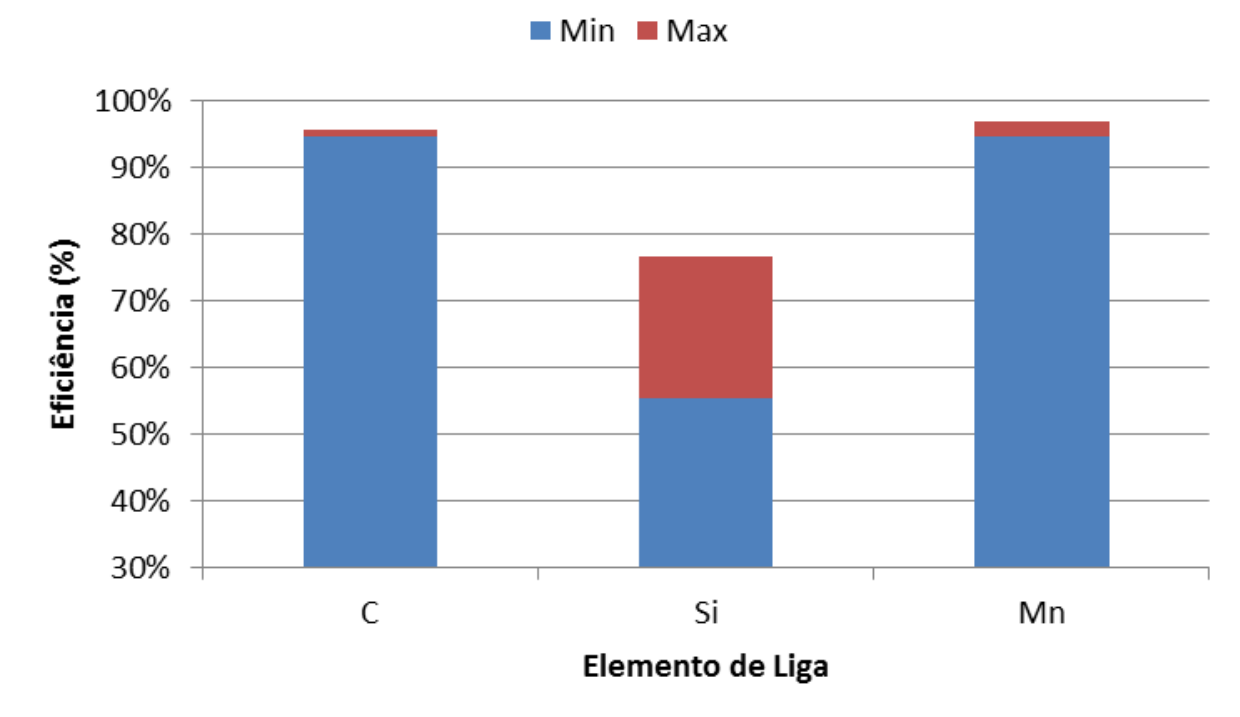

Figura 16: Eficiência Metálica Considerada a Remoção Dupla de Escória

\section{CONCLUSÕES}

Os resultados do modelo de carregamento desenvolvido neste artigo se apresentaram tecnicamente equivalentes aos encontrados na literatura para o cenário 1 e tecnicamente equivalentes e mais econômicos para o cenário 2. Além disso, eles apresentam custos menores quando comparados com as práticas da empresa, com uma economia relativa média de $(26,67 \pm 7,10) \%$ para a liga SAE 1045 . Os indicadores $\zeta_{j}$ e $\zeta_{j}^{F e}$ apresentam diferença estatística entre os resultados da empresa e deste modelo, isto é, gastou-se menos com elementos de liga e mais com ferro no seu carregamento. O indicador $\tau_{j}$ aponta que se supriu de maneira equivalente as necessidades de elementos de liga da liga SAE 1045 tanto com a metodologia da empresa quanto com a deste modelo. Os resultados de eficiência metálica do modelo são maiores que os valores de processo encontrados pela empresa, porém ao se estimar seus novos valores considerando a renovação intencional da escória durante o processo, seus resultados se aproximam dos mesmos valores de eficiência metálica do processo.

\section{AGRADECIMENTOS}

Os autores deste artigo agradecem à CAPES pela bolsa de Mestrado e à empresa Ecoplan Aços Especiais pelo apoio e dados compartilhados.

\section{BIBLIOGRAFIA}

[1] CARVAlHO, J. L., ASSIS, P. S., FIGUEIRA, R. M., et al., Dados Termodinâmicos para Metalurgistas, Belo Horizonte, Universidade Federal de Minas Gerais, 1977.

[2] CAMPOS, V. F. Tecnologia de Fabricação do Aço Líquido, v.1, 3 ed., Belo Horizonte, [s.n], 1985. 
[3] TERANO, T., KIYOJI, A., MICHIO, S., Fuzzy System Theory and its Applications, Boston, Academic Press, 1992.

[4] ZIÓLKOWSKI, E., WRONA, R.,"Using Fuzzy Optimisation Method in Calculation of Charge Burden to Correct the Chemical Composition of Metal Melt", Archives of Foundry Engineering, v. 7, n. 3, pp. 183-186, 2007.

[5] SAKALLI, U. S., BAYKOÇ, O. F., “An Optimization Approach for Brass Casting Blending Problem under Aleatory and Epistemic Uncertainties”, International Journal of Production Economics, v. 133, n.2, pp.708-718, 2011.

[6] RONG, A., LAHDELMA, R., "Fuzzy Chance Constrained Linear Programming Model for Optimizing the Scrap Charge in Steel Production”, European Journal of Operational Research, v. 186, n.3, pp. 953-964, 2008.

Sons, 2009.

[7] MONTGOMERY, D. C., Design and Analysis of Experiments, 7 ed., New York, John Wiley \&

\section{ANEXOS} trabalho.

A seguir se encontra descrito o uso da ferramenta ANOVA na análise estatística dos resultados deste

\subsection{Ferramenta ANOVA}

Seja um experimento com ' $a$ ' tratamentos, ou níveis de experimentação, e ' $n$ ' observações ' $y$ ' de um único fator. Os dados podem ser visualizados conforme a Tabela 4.

Tabela 3: Valores experimentais observados para ' $a$ ' tratamentos e ' $n$ ' observações

\begin{tabular}{|c|c|c|c|c|c|c|}
\hline Tratamento & \multicolumn{4}{|c|}{ Observações } & Totais & Médias \\
\hline 1 & $y_{11}$ & $y_{12}$ & $\cdots$ & $y_{1 n}$ & $y_{1}$. & $\overline{y_{1}}$. \\
\hline 2 & $y_{21}$ & $y_{22}$ & $\cdots$ & $y_{2 n}$ & $y_{2}$. & $\overline{y_{2}}$. \\
\hline & $\ldots$ & $\ldots$ & $\ldots$ & $\ldots$ & $\ldots$ & $\ldots$ \\
\hline$a$ & $y_{a 1}$ & $y_{a 2}$ & $\ldots$ & $y_{a n}$ & $y_{a}$. & $\overline{y_{a}}$. \\
\hline & & & & & y.. & $\overline{\mathrm{y} . .}$ \\
\hline
\end{tabular}

Deseja-se testar a hipótese $H_{0}$ descrita na equação (25), em que as médias ' $\overline{y_{l}}$ ' dos ' $a$ ' tratamentos são iguais. Havendo diferença estatística entre as médias, a hipótese $H_{1}$ é verdadeira.

$H_{0}: \overline{y_{1}}=\overline{y_{2}}=\cdots=\overline{y_{\mathrm{a}}}$.

$H_{1}: \overline{y_{1}}, \neq \overline{y_{\mathrm{j}}} . \quad \forall(i, j)$

As fórmulas para cálculo da função de probabilidade $F$ se encontram descritas na Tabela 5, abaixo.

Tabela 5: Formulário para Ferramenta ANOVA

\begin{tabular}{|c|c|c|c|c|}
\hline & Soma Quadrática & $\begin{array}{l}\text { Graus de } \\
\text { Liberdade }\end{array}$ & Média Quadrática & $\boldsymbol{F}$ \\
\hline Entre Tratamentos & $S S_{\text {Trat }}=\frac{1}{n} \sum_{i=1}^{a} y_{i .}^{2}-\frac{y_{. .}^{2}}{a n}$ & $a-1$ & $M S_{\text {Trat }}=\frac{S S_{\text {Trat }}}{a-1}$ & $\frac{M S_{\text {Trat }}}{M S_{E}}$ \\
\hline $\begin{array}{l}\text { Dentro de } \\
\text { Tratamentos }\end{array}$ & $S S_{E}=S S_{T}-S S_{T r a t}$ & $a n-\boldsymbol{a}$ & $\boldsymbol{M} \boldsymbol{S}_{E}=\frac{S \boldsymbol{S}_{E}}{a n-\boldsymbol{a}}$ & \\
\hline Total & $S S_{T}=\sum_{i=1} \sum_{j=1} y_{i j}^{2}-\frac{y_{. .}}{a n}$ & $a n-\mathbf{1}$ & & \\
\hline
\end{tabular}

